\title{
Chronic pramipexole treatment increases tolerance for sucrose in normal and ventral tegmental lesioned rats
}

\author{
David Dardou ${ }^{1}$, Carine Chassain ${ }^{2}$ and Franck Durif ${ }^{1,3}$ \\ ' EA7280 NPSY-Sydo, Université d'Auvergne, Clermont-Ferrand, France \\ 2 IRM-Hopital Gabriel Montpied, Clermont-Ferrand, France \\ ${ }^{3}$ Service de Neurologie A, Hopital Gabriel Montpied, Clermont-Ferrand, France
}

Edited by:

Andrew C. McCreary, Brains

On-Line, Netherlands

Reviewed by:

David Tattersall, GlaxoSmithKline,

China

Ciaran J. Faherty, Cadence

Pharmaceuticals, USA

\section{*Correspondence:}

David Dardou, Faculté de Médecine,

EA7280 NPSY-Sydo, Université

d'Auvergne (UdA), 28 place Henri

Dunant, BP38, 63001

Clermont-Ferrand, France

e-mail:david.dardou@udamail.fr
The loss of dopamine neurons observed in Parkinson's disease (PD) elicits severe motor control deficits which are reduced by the use of dopamine agonists. However, recent works have indicated that D3-preferential agonists such as pramipexole can induce impulse control disorders (ICDs) such as food craving or compulsive eating. In the present study, we performed an intermittent daily feeding experiment to assess the effect of chronic treatment by pramipexole and VTA bilateral lesion on tolerance for sucrose solution. The impact of such chronic treatment on spontaneous locomotion and spatial memory was also examined. Changes in sucrose tolerance could indicate the potential development of a change in food compulsion or addiction related to the action of pramipexole. Neither the bilateral lesion of the VTA nor chronic treatment with pramipexole altered the spontaneous locomotion or spatial memory in rats. Rats without pramipexole treatment quickly developed a stable intake of sucrose solution in the $12 \mathrm{~h}$ access phase. On the contrary, when under daily pramipexole treatment, rats developed a stronger and ongoing escalation of their sucrose solution intakes. In addition, we noted that the change in sucrose consumption was sustained by an increase of the expression of the Dopamine D3 receptor in the core and the shell regions of the nucleus accumbens. The present results may suggest that long-term stimulation of the Dopamine D3 receptor in animals induces a strong increase in sucrose consumption, indicating an effect of this receptor on certain pathological aspects of food eating.

Keywords: sucrose tolerance, ventral tegmenta area, Parkinson's disease, dopamine agonist, pramipexole, food compulsion

\section{INTRODUCTION}

The loss of dopamine (DA) producing neurons observed in Parkinson's disease (PD) elicits severe motor control deficits including rest tremor, bradykinesia, and rigidity. While dopamine replacement therapy (DRT) reduces motor symptoms, a growing number of studies have shown that the use of DRT, in particular dopamine agonists, induces impulse control disorder (ICD) such as dopamine dysregulation syndrome, hypersexuality, pathological gambling, compulsive shopping, and disturbance of eating behavior (Courty et al., 1997; Gallagher et al., 2007; Voon and Fox, 2007; Merims and Giladi, 2008; Weintraub et al., 2010). Clinical studies have shown that PD patients occasionally develop food craving or compulsive eating disorder (Nirenberg and Waters, 2006; Miwa and Kondo, 2008; Rieu et al., 2011). Such abnormal behavior generally induces a significant, undesired weight gain that can disturb the PD patient's quality of life.

Progressive loss of dopaminergic neurons leads to the denervation of both the nigro-striatal pathway (around $80 \%$ ) from the substantia nigra (SN) and the mesocorticolimbic system (around $40 \%$ ) from the ventral tegmental area (VTA) (German et al., 1989). Although the loss of dopaminergic neurons in the $\mathrm{SN}$ is directly linked to the motor and apathy symptoms observed in PD patients and animal models (Dagher and Robbins, 2009; Drui et al., 2013), the relation between the lesion of the dopaminergic brain region and ICD remains unclear. Although the lesion of the VTA in Parkinsonian patients is rather limited, the loss of dopaminergic neurons in this brain region could account, at least partially, for the development of ICD in PD patients under DRT. Indeed, the main dopaminergic afferent projections coming from the VTA are directed to the nucleus accumbens (Acb). The dopamine arising from these afferent projections acts on the Dopamine D2 and D3 receptors in the Acb. This nucleus plays a key role in the regulation of the mesocorticolimbic system (Robinson and Berridge, 2001) due to its place in the brain's reward circuit.

Dopamine agonists, particularly pramipexole (PPX), induces conditioned place preference in normal animals as well as those with 6-OHDA lesions of the striatum or SN (Papp, 1988; Riddle et al., 2012; Zengin-Toktas et al., 2013). Interestingly, it was observed that sham-operated animals need a higher dose of PPX to induce conditioned place preference compared to rats with dopaminergic lesions in the striatum, pointing to potentially higher sensitivity to the reward properties of PPX in rats 
with lesions (Riddle et al., 2012). In addition, recent works have found that rats with lesions of the posterior region of the VTA develop a conditioned place preference induced by D2 and D3 agonists (Ouachikh et al., 2013), while lesion of the anterior region of the VTA disrupts the ability of rats to learn conditioned place preference (Ouachikh et al., 2014). Although the conditioned place preference paradigm appears to be limited to the study of motivational aspects linked to ICD, such data could indicate possible interaction between dopamine agonist treatment and lesion of the VTA region, potentially leading to a change in motivated behaviors. In addition, recent clinical studies from Thobois et al. (2010, 2013) have shown that non-motor symptoms (apathy, depression, and anxiety) was more expressed in patients with enhanced denervation of mesocorticolimbic pathway.

If VTA denervation contributes to CPP learning in rodents and to apathy, depression, and anxiety in PD patients, it is possible that it could also participate in certain aspects of impulsive/compulsive disorders. To address this question, we chose in the present work to investigate the effect of VTA lesion and dopamine agonist treatment in a model of compulsive food disorder, i.e., intermittent daily feeding. Intermittent daily feeding is a well-described behavioral animal model of food addiction or binge eating in which rats develop tolerance to sucrose solution by successive day-to-day access as well as signs of withdrawal when access to sucrose is removed (Avena et al., 2008a,b; Avena, 2010). Using this behavioral approach, we investigated the effect of the lesion of the VTA region and chronic PPX administration on sucrose solution tolerance, which preferentially activates the D3 receptor at low doses (Collins et al., 2005). Interestingly, an increase of D3 mRNA expression in the neurons of the direct striatonigral pathway was observed in rats with 6-OHDA lesion under chronic administration of L-Dopa (Bordet et al., 2000). Furthermore, this receptor is implicated in certain aspects of drug dependence such as drug seeking and relapse (Le Foll et al., 2005). Blockade of the D3 receptor by a selective antagonist has been reported to transiently alleviate craving to nicotine in smoking addicts (Mugani et al., 2013) and to disrupt morphine triggered cocaine induced CPP in rats (Rice et al., 2013).

The aim of the present study was to assess the effect of pramipexole and bilateral VTA lesions in rats on tolerance for sucrose solution using a model of daily intermittent sucrose access, which is considered to mimic aspects of compulsive food eating in humans. In addition, we also explored the effect of such chronic treatment on motor, cognitive, and anxiety-like traits. In addition, change in D3 expression in the Acb was explored.

\section{MATERIALS AND METHODS SUBJECTS}

Forty-five male Sprague Dawley rats (Charles River, Les Oncins, France) weighing 200-225 g were housed individually. Animals had access ad libitum to food and water before the behavioral studies were started. All the experiments were carried out in conformity with the European Committee Council Directive of 24 November 1986 (86/609/EEC) and were approved by the local ethical committee (CEMAAuvergne).

\section{BILATERAL 6-OHDA LESION AND PRAMIPEXOLE TREATMENT}

Animals were anesthetized by an i.p. injection of mixture of ketamine $(60 \mathrm{mg} / \mathrm{Kg})$ and xylazine $(20 \mathrm{mg} / \mathrm{Kg})$ and treated with desipramine $(25 \mathrm{mg} / \mathrm{Kg}$, i.p., Sigma France) $30 \mathrm{~min}$ before 6-OHDA injection. Bilateral lesions of the VTA were performed by micro injection of 6-OHDA $(2 \mu \mathrm{g} / \mu \mathrm{L}$, dissolved in $\mathrm{NaCl} 0.9 \%$ with $0.01 \%$ of ascorbic acid solution, Sigma France) at the following coordinate: AP: $-5.2 \mathrm{~mm}$; Lat: $\pm 0.8 \mathrm{~mm}$; DV: $-7.6 \mathrm{~mm}$ from the bregma at a speed of $0.125 \mu \mathrm{L} / \mathrm{min}$ for a total of $1 \mu \mathrm{l}$ injected on each side. The syringe was left in position for at least $10 \mathrm{~min}$ to reduce diffusion along the needle tract. Sham-operated animals received the same procedure with the infusion of the vehicle ( $\mathrm{NaCl} 0.9 \%$ with $0.01 \%$ of ascorbic acid solution, Sigma France). Animals had a 2-week rest period to regain normal home caged behaviors and to stabilize the 6-OHDA lesion.

Two groups of rats (sham and 6-OHDA lesioned rats) were treated daily with an s.c. injection of pramipexole (PPX, Sigma France) at a dose of $0.1 \mathrm{mg} / \mathrm{kg} /$ day (dissolved in $0.9 \% \mathrm{NaCl}$ ). PPX was administered every morning at 7 a.m. by subcutaneous injection from the 17th experimental day (Figure 1) to the end of the experiment (day 49, so rats were treated for 32 days). Two other control groups (sham and 6-OHDA lesioned rats) were s.c. injected daily with $0.9 \% \mathrm{NaCl}$ solution. Lesioned and sham animals treated with PPX are referred to as L-PPX $(n=12)$ and Sh-PPX $(n=10)$, respectively. The control groups are referred to as $\mathrm{L}(n=12)$ and $\mathrm{Sh}(n=11)$ for the lesioned and sham groups, respectively. The treated animals received an injection once a day at the same time ( 7 a.m.) and at least $2 \mathrm{~h}$ before behavioral tasks took place.

\section{BEHAVIORAL TESTING}

After a recovery period, all the animals completed the following sequence of behavioral tests (Figure 1). Animals were evaluated in open field box and alternation in a Y maze in order to estimate spontaneous locomotor activity and spatial memory before and after 1 week of PPX treatment, respectively. The anxiety level of animals was measured the day before and 2 days after the last day of access to the $10 \%$ sucrose solution.

\section{$Y$ maze}

Alternation in the $\mathrm{Y}$ maze made of gray plastic was performed under soft light (30-35 lux). It consisted of three identical branches ( $45 \mathrm{~cm}$ long, $13 \mathrm{~cm}$ wide, and $20 \mathrm{~cm}$ high) which diverged at an angle of $120^{\circ}$ from the central point. Animals were placed in the start branch and freely explored the maze for $5 \mathrm{~min}$. The orders of the visits were noted by the observer. The maze was cleaned with hot water and ethanol between each run. Alternation was determined from successive entries (four paws inside the branch) of the three branches by overlapping triplet sets in which three different branches were entered (Taghzouti et al., 1986; Pioli et al., 2008). The alternation score was obtained by dividing the number of alternations by the number of alternation opportunities (the total branch entries minus 2).

\section{Open field}

Spontaneous motor activity was measured in an open field box ( $75 \mathrm{~cm}$ long, $75 \mathrm{~cm}$ wide, and $45 \mathrm{~cm}$ high) placed under 


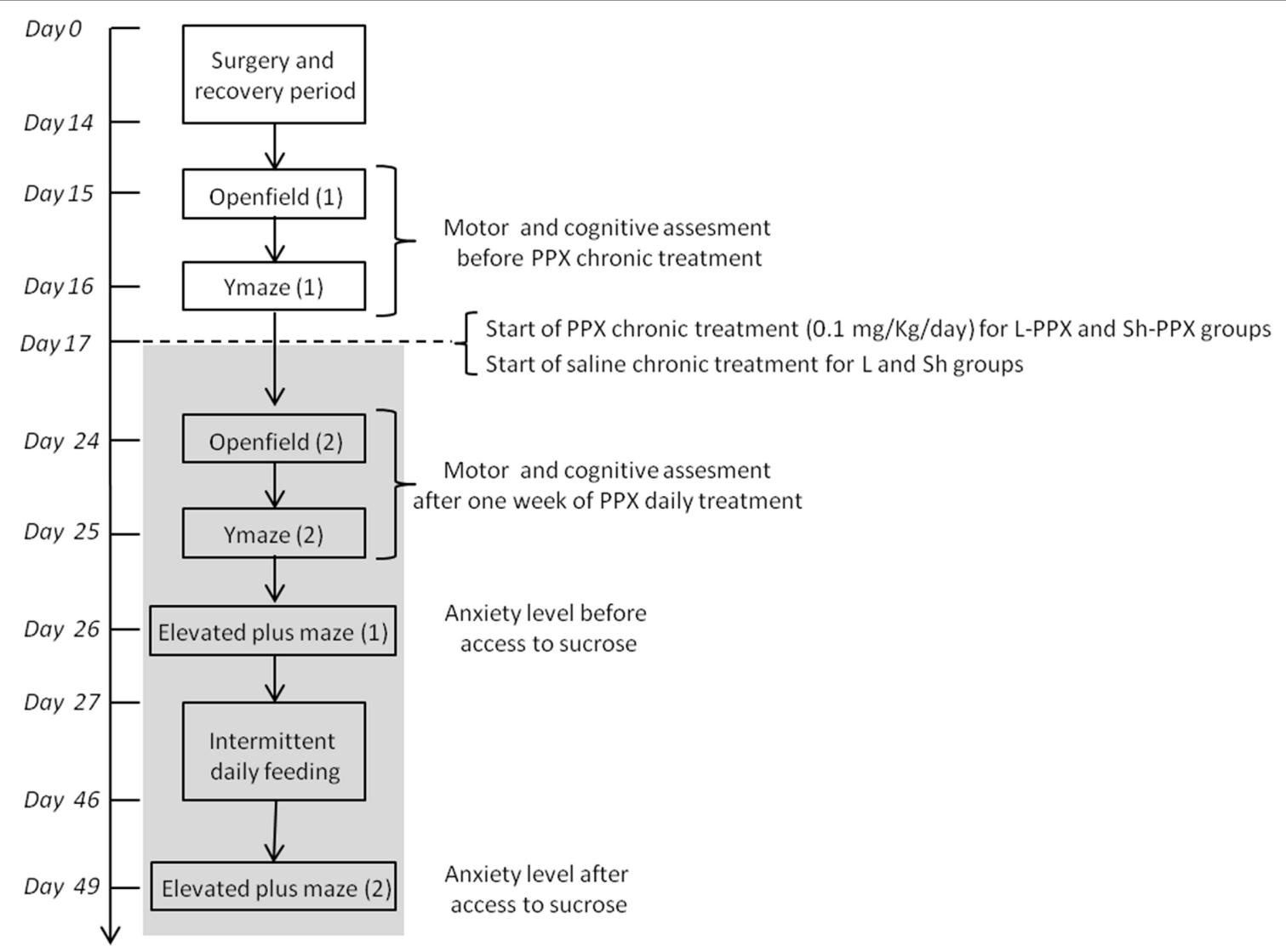

FIGURE 1 | Time schedule of behavioral experiments. After a period of acclimation to animal house, rats received 6-OHDA or vehicle solution injection in the VTA. After the recovery period of 2 weeks, all animals were evaluated for spontaneous locomotion and spontaneous alternation in $Y$ maze on 2 days. After the first evaluation, chronic PPX treatment starts with daily administration of $0.1 \mathrm{mg} / \mathrm{kg} /$ day of PPX by s.c. injection and last until the end of the experiment (light gray box). A second evaluation of spontaneous locomotion and spontaneous alternation in $Y$ maze was performed after 1 week of PPX treatment. The anxiety level of animal was measured using elevated plus maze, the day before and 2 days after the last day of the $10 \%$ sucrose solution access. Intermittent daily feeding was assessed during 19 consecutive days. a camera used to record each trial under soft lighting (3035 lux). Animals were placed in the center of the box and were allowed to move freely during $15 \mathrm{~min}$. All sessions were video-recorded and analyzed with EthoVision XT 8.5 (Noldus, Netherlands). The open field arena was then cut in 16 squares of identical size for analysis. The mean distance run (in $\mathrm{cm}$ ) and number of squares crossed and the numbers of rearings were analyzed.

\section{Elevated plus maze}

Anxiety behavior was assessed using an elevated plus maze $(50 \mathrm{~cm}$ long, $10 \mathrm{~cm}$ wide, $10 \mathrm{~cm}$ high for the protected arms, $52 \mathrm{~cm}$ from the ground) under soft illumination (30-35 lux) and a camera was used to record all the trials. Rats were placed in the center of the device and were free to move for $5 \mathrm{~min}$. The time spent in open arm ratio (OTR) was calculated by dividing the times spent in the open arm by the time spent in the open and closed arms. OTR was used as the measure of anxiety as well as the number of entries in the non-protected arms in comparison to the protected one. The experiment was analyzed using EthoVision XT 8.5 (Noldus, Netherlands).

\section{Intermittent daily feeding}

All experimental groups were placed under daily alternation for 19 days. During this period, animals received a drinking bottle containing a $10 \%$ sucrose solution at the start of the dark phase $(12 \mathrm{~h})$. The present protocol was adapted from the studies of Avena et al. (2008a,b) to our animal house condition. The bottles containing the $10 \%$ sucrose solution was given to rats immediately at the start of the dark period (7 p.m.) and removed at the start of the light phase (7 a.m.). Animals had access to tap water only during the light phase. The amount of sucrose solution (in g.) was noted by weighing the bottle before and after the dark period. Food was accessible ad libitum throughout the experiment.

\section{Detection of tyrosine hydroxylase and Dopamine $D 3$ receptor expression}

After the completion of the behavioral evaluations, rats were killed by chloral hydrate injection. The brains of the animals were collected and post-fixed for $24 \mathrm{~h}$ in PFA $4 \%$, before being processed for paraffin embedded sections. Paraffin blocs were then 
serially cut into $7 \mu \mathrm{m}$ thick sections (first slice chosen randomly) and mounted on glass slides.

Detection of tyrosine hydroxylase $(\mathrm{TH})$ was used to determine the extent of the 6-OHDA lesion. Paraffin was removed by successive baths of xylene, ethanol and bathing in hot citrate buffer for $25 \mathrm{~min}$. After cooling at room temperature, the slides were rinsed in tris-phosphate buffer $0.1 \mathrm{M}$ (TBS $0.1 \mathrm{M}$ ) and endogeneous peroxydases were quenched with TBS $0.1 \mathrm{M}$ with $5 \%$ $\mathrm{H}_{2} \mathrm{O}_{2}$ for $30 \mathrm{~min}$. Sections were then incubated with anti-TH antibody (1/5000, rabbit, AB152 Milipore USA) diluted in DAKO REAL antibody diluents overnight at room temperature. The next day, the presence of the epitope was revealed using the DAKO LSAB and HRP system (DAKO Real, DAKO France). The reaction allowed localizing the expression of $\mathrm{TH}$ by brown labeling on the slides. In order to reduce the differences in background labeling between all the slides, all the slides from the experiments were processed at the same time for $\mathrm{TH}$ immunohistochemistry under the same laboratory conditions. TH-positive neurons were counted using ImageJ software on 12 slices from the anterior to posterior axis of the brain. The numbers of positive cells in both VTA and SN were estimated with the method described by Paillé et al. (2007).

Slices containing the accumbens and striatum at the level around $+2.16 \mathrm{~mm}$ from Bregma were processed for the detection of Dopamine D3 receptor expression. After pretreatment, the slices were incubated at room temperature with TBS solution containing 3\% normal goat serum (NGS, Jackson ImmunoResearch USA) for $1 \mathrm{~h}$. After rinsing, the slices were incubated overnight at $4^{\circ} \mathrm{C}$ with a solution of TBS containing $1 \%$ NGS and antidopamine D3 receptor antibody (1/200, rabbit polyclonal, sc9114, Santa Cruz USA). The next day, the slides were rinsed using TBS and incubated with secondary antibody (goat anti rabbit alexa 488, Jackson ImmunoResearch USA) for $1 \mathrm{~h}$ at room temperature. The slides were rinsed and mounted using Dako fluorescent mounting medium and kept in the dark at $4^{\circ} \mathrm{C}$ before analysis. In order to reduce the differences in background labeling between all the slides, all the slides from the experiments were processed at the same time for D3R immunohistochemistry under the same laboratory conditions. Observation of the fluorescent labeling was done using a Zeiss microscope and AxioVision software. The number of positive cells was counted in $100 \mu \mathrm{m}^{2}$ square windows, positioned at the same place using a brain atlas. In the Acb, one window was used for the core region and one window for the shell region. The numbers of positive cells was noted as the mean number of cells per $100 \mu \mathrm{m}^{2}$.

\section{DATA ANALYSIS}

All behavioral data were expressed as mean \pm S.E.M. for each experimental group and analyzed using a Two-Way repeatedmeasures analysis of variance (ANOVA) with lesion (two levels, lesioned and sham) and treatment (two levels, PPX or saline) as within subject factors. When appropriate, data were compared using the Tukey-Kramer post-hoc test for multiple comparisons.

For sucrose consumption, the intake of sucrose was also expressed using the area under curves (A.U.C.). The A.U.C. was obtained by summing the squares under the curves for each group using the trapezoidal rule as followed: $\Sigma$ [(VTtime X - VTtime 0) (time $\mathrm{X}$ - time X-1)]. The A.U.C. values, the mean number of $\mathrm{TH}$ positive cells and the mean number of D3 positive cells were analyzed by a One-Way ANOVA with the experimental groups (four levels, L or L-PPX or Sh or Sh-PPX) as the factor and, when appropriate, data were compared using Tukey-Kramer post-hoc tests for multiple comparisons. Statistical analysis was performed with SAS 9.1 software (SAS institute Inc., USA).

\section{RESULTS}

\section{BILATERAL PARTIAL LESIONS OF THE VTA}

The effect of the bilateral micro-injection of 6-OHDA revealed by $\mathrm{TH}$-immunoreactivity indicated a discrete pattern of dopamine denervation centered on the VTA. One-Way ANOVA showed a significant effect of the groups for the VTA region only $\left[F_{(3,43)}=\right.$ 57.66; $p<0.0001]$. In this region, the mean number of TH positive cells was significantly ( $p<0.05$ ) lower in L and L-PPX groups compared to Sh and Sh-PPX groups (Figure 2A). A decrease of $36 \%$ and $33 \%$ of $\mathrm{TH}$-positive cell density was observed in $\mathrm{L}$ and L-PPX groups respectively compared to sham rats. Lesion of the VTA appeared to be restricted to the medial part of the VTA in L and L-PPX rats (Figure 2B), a region known to mainly project to the accumbens nucleus. On the contrary, the decrease in the SN remained very low (less than $2 \%$ ) and no significant differences were observed between groups.

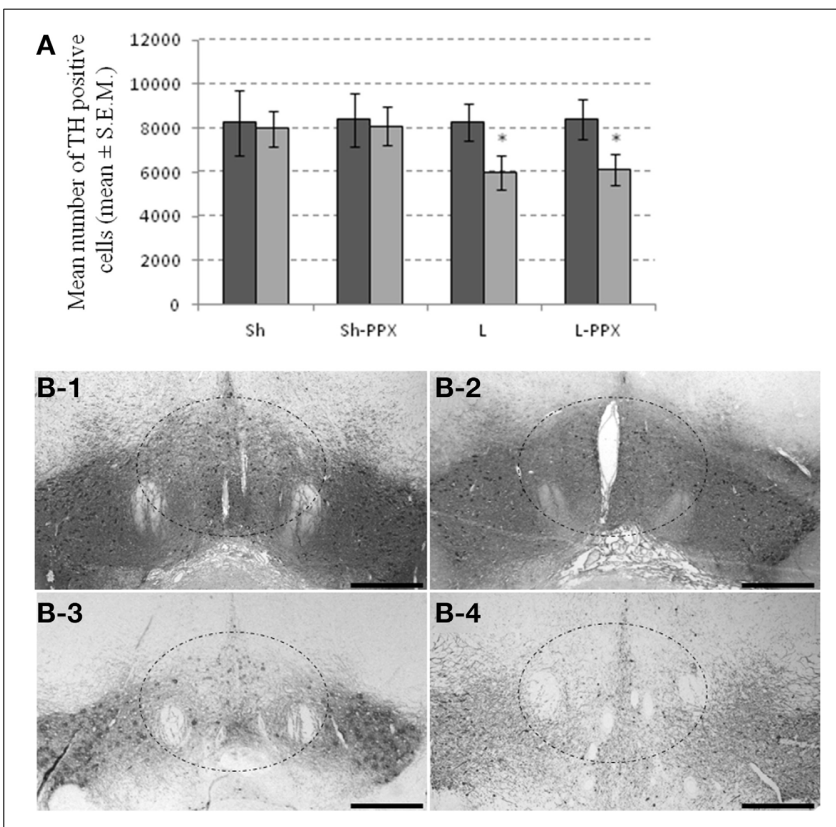

FIGURE 2 | Partial bilateral lesion of VTA by 6-OHDA micro-injection. The injection of 6-OHDA produced a decrease in the number of TH-positive cells within the VTA of lesioned rats (A). The dark gray bar represented the number of positive cells counted in the SN, while light gray bar represented the number of positive cells counted in the VTA region. Microphotographs illustrated the dopamine denervation following 6-OHDA injection in Sh (B-1), Sh-PPX (B-2), L (B-3), and L-PPX (B-4). The dash line square represents the region where lesion is the most representative. Black scales bars represent $100 \mu \mathrm{m}$. Microphotographs were taken around $-5.28 \mathrm{~mm}$ from the Bregma. 


\section{BEHAVIORAL RESULTS}

\section{Effect of the daily PPX treatment}

Spontaneous locomotor activity in the open field. The TwoWay ANOVA with repeated measures did not show a significant effect of factors on the mean distance run in the open field arena (Figure 3A) or the amount of rearing (Figure 3B). Overall, the PPX daily administration had no impact on the locomotor activity of rats, whether they were lesioned or not.

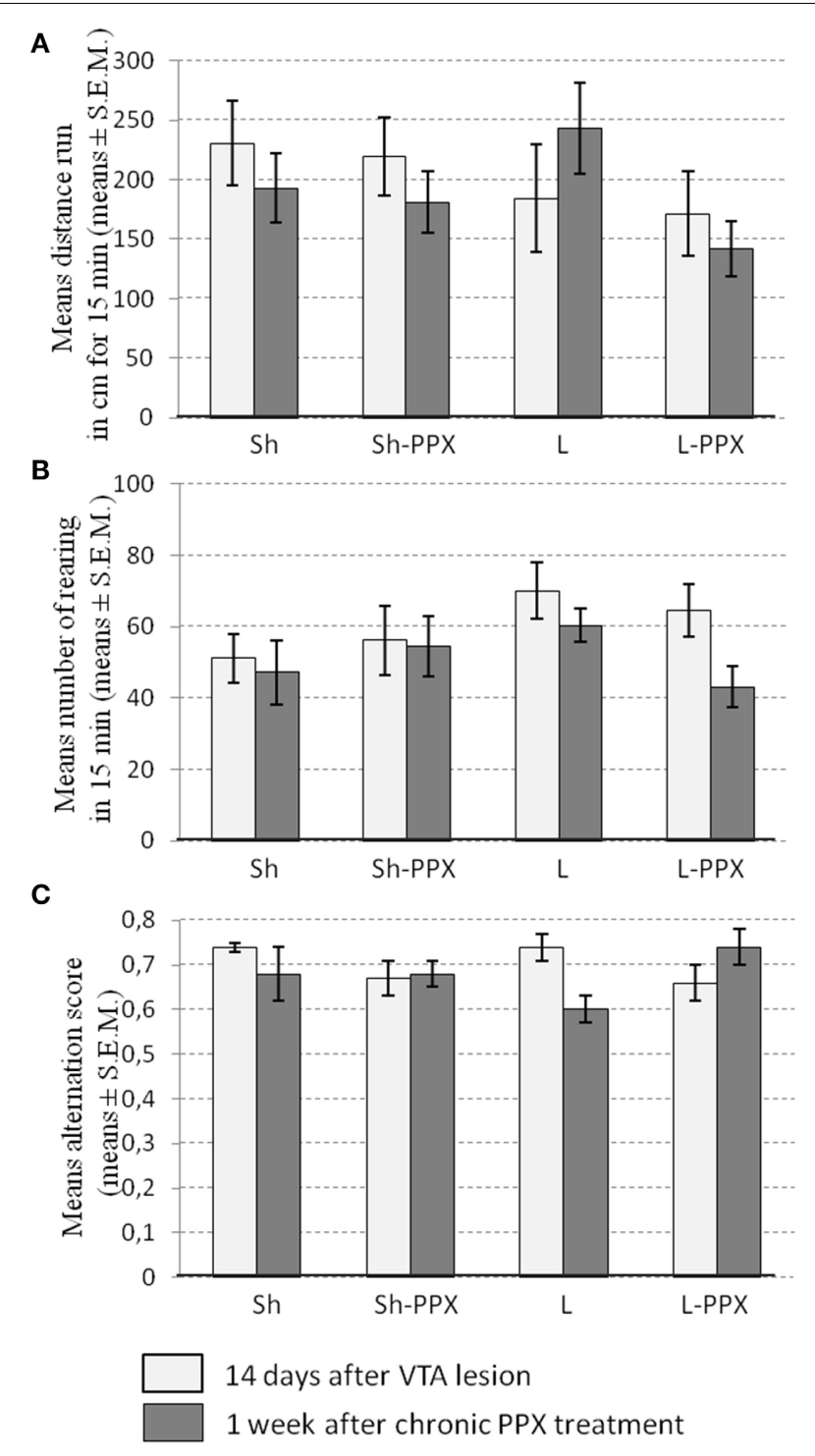

FIGURE 3 | Daily administration of pramipexole (PPX) has no effect on spontaneous locomotors activity and spontaneous alternation $Y$ maze. Spontaneous locomotor activity (A) and rearing (B) were not affected by the VTA lesion (white bars) or by 1 week of daily administration of PPX (gray bars) whatever the experimental group considered. The alternation score measure in $Y$ maze (C) was not modified by neither the lesion (white bars) nor 1 week of daily PPX administration (gray bars). Sh, Sham animals; Sh-PPX, sham animals with daily pramipexole treatment $(0.1 \mathrm{mg} / \mathrm{kg} /$ day); L, VTA lesioned rats; L-PPX, VTA lesioned rats with daily pramipexole treatment $(0.1 \mathrm{mg} / \mathrm{kg} / \mathrm{day})$.
Spontaneous alternation in the Y maze. The Two-Way ANOVA with repeated measures indicated significant interaction between repetition and treatment $\left[F_{(1,43)}=8.34 ; p<0.05\right]$ without significant difference in the post-hoc tests. We noted that the alternation score in Sh and L rats appeared to decrease slightly 1 week after daily PPX treatment (Figure 3C), whereas its increased slightly in L-PPX rats, although these differences were not significant.

\section{Intermittent daily feeding}

The daily intake of a $10 \%$ sucrose solution for the $12 \mathrm{~h}$ of the dark period escalated over the days, irrespective of the experimental group considered (Figure 4A). The Two-Way ANOVA for repeated measures indicated a significant effect of treatment $\left[F_{(1,43)}=8.56 ; p<0.05\right]$, a significant effect of repetition $\left[F_{(18,774)}=26.8 ; p<0.0001\right]$ and an interaction between repetition and treatment $\left[F_{(18,774)}=7.48 ; p<0.0001\right]$. An additional analysis using a One-Way ANOVA with day as factor for each group indicated an effect only in L-PPX and Sh-PPX [respectively $F_{(18,246)}=9.15 ; p<0.0001$ and $\left.F_{(18,208)}=7.91 ; p<0.0001\right]$.

The day-to-day sucrose intake slowly escalated from the first to the tenth day for all experimental groups. In the L group, although rats drank less sucrose solution than the other groups (no significant difference), their intake escalated slowly. On the 14th day, a clear distinction appeared between groups. Since the sucrose solution intake remained stable for the rats receiving saline injections, those treated with pramipexole continued to increase their sucrose intake slowly. The sucrose consumption of rats treated with pramipexole was significantly $(p<0.05)$ higher compared to the saline treated groups on the 18th and 19th days, indicated by the Tukey post-hoc test.

Interestingly, in the L-PPX and Sh-PPX groups, the shapes of the sucrose intake escalation curves were similar with a continuous increase of sweet solution intake. From the 11th day to the 13th day, the sweet solution intake for the L-PPX group continued to increase, while it remained mostly constant for the other groups. On day 14 to day 19, the volume of $10 \%$ solution consumed by L-PPX rats was significantly ( $p<0.05$ ) higher compared to L rats. For the Sh-PPX group, sucrose solution intake was significantly $(p<0.05)$ higher compared to L rats, but was never significantly different from Sh rats. In addition, the A.U.C. analysis showed a significant group effect $\left[F_{(3,43)}=2.80 ; p=0.05\right]$ in a One-Way ANOVA with group. Post-hoc tests indicated that the A.U.C. was significantly $(p=0.05)$ higher for the L-PPX group compared to the L (Figures 4B,C).

\section{Change in anxiety after removing sucrose access}

Change in OTR. The Two-Way ANOVA for repeated measures indicated an effect of repetition $\left[F_{(1,43)}=28.83 ; p<0.0001\right]$, of treatment $\left[F_{(1,43)}=17.43 ; p<0.0001\right]$ and a significant interaction between these two factors $\left[F_{(3,43)}=20.03 ; p<0.0001\right]$ on the OTR ratio measure before and after access to sucrose. Before the initiation of intermittent access to the sucrose solution, the OTR ratio was significantly $(p<0.001)$ lower in the L-PPX groups compared to all the other experimental groups (Figure 5A). In addition, we also noted that this ratio in Sh-PPX rats was significantly $(p<0.05)$ lower compared to that of Sh 


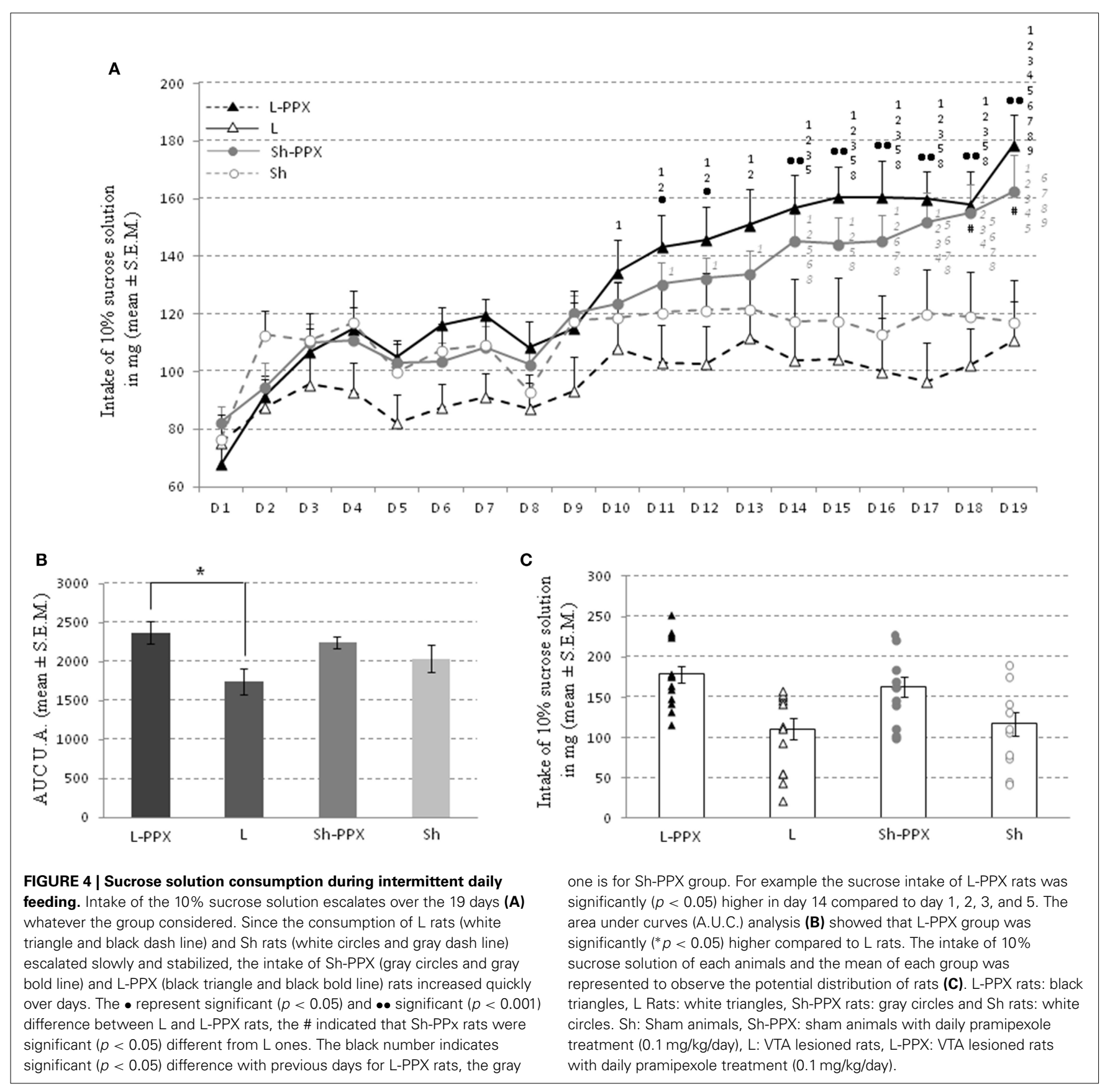

rats. When the OTR was evaluated again 2 days after removing access to the sucrose solution, we noted a significant $(p<0.05)$ decrease in this ratio in all the animal groups compared to the value before the period of access to sucrose. Nevertheless, we did not observe significant differences in this ratio between the experimental groups when evaluated after the withdrawal of access to sucrose.

Number of entries in the open arms. The frequency of entry into the open arms was also analyzed as an index of anxiety. The Two-Way ANOVA for repeated measures indicated a significant effect of the treatment factor $\left[F_{(1,43)}=4.28 ; p<0.05\right]$ and of repetition $\left[F_{(1,43)}=70.64 ; p<0.0001\right]$. Tukey-Kramer post-hoc test indicated that saline treated animals were significantly $(p<$ 0.05) different from the PPX treated ones (Figure 5B). The decrease of the frequency of entrance between before and after sucrose access was around 58\% in Sh animals, 55\% in Sh-PPX animals, 50\% in L animals, and 64\% in L-PPX animals, but no significant differences were observed between groups.

\section{CHANGE OF DOPAMINE D3 RECEPTOR EXPRESSION IN THE ACCUMBENS NUCLEUS REGION}

Analysis of D3R immunoreactivity in the core (Figures 6A,C) and shell (Figures 6B,D) regions of the Acb by a One-Way ANOVA 


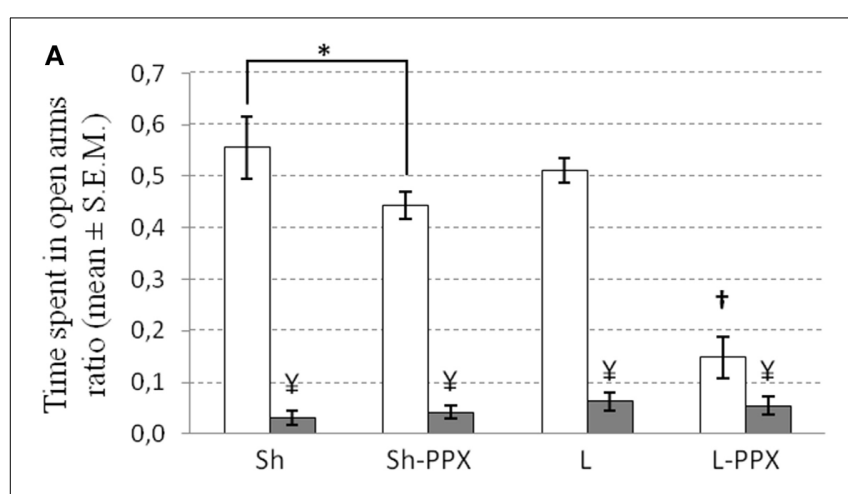

B

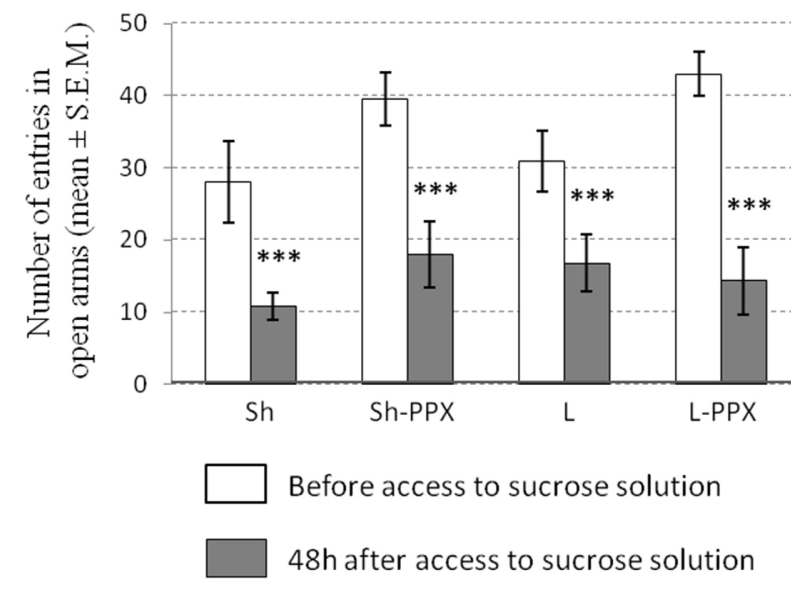

FIGURE 5 | Effect of sucrose access removal on anxiety measured by elevated plus maze. (A) Time spent in open arm ratio (OTR) for each experimental groups before intermittent daily access (white bars) and after 2 days of removing access to sucrose solution (gray bars). OTR was significantly lower in L-PPX compared to all other groups before access to sucrose $\left({ }^{\dagger} p<0.001\right)$. OTR was also significantly lower in Sh-PPX compared to Sh one $\left(^{*} p<0.05\right)$. When access to sucrose was removed to animals, we noted a significant decrease of the OTR in all experimental groups compared to value before access to sucrose solution ( $¥ p<0.05$ ). (B) The number of entries in open arms was significantly decreased $(* * * p<0.0001)$ when compared before having access to $10 \%$ sucrose solution period (white bars) to after removal access period (gray bars). Sh, Sham animals; Sh-PPX, sham animals with daily pramipexole treatment $(0.1 \mathrm{mg} / \mathrm{kg} / \mathrm{day}) ; \mathrm{L}$, VTA lesioned rats; L-PPX, VTA lesioned rats with daily pramipexole treatment $(0.1 \mathrm{mg} / \mathrm{kg} /$ day).

indicated a significant effect of the experimental group for these two brain regions [core: $F_{(3,24)}=8.17 ; p<0.05$; shell: $F_{(3,24)}=$ 4.77; $p<0.05$. Post-hoc tests indicated that the number of positive cells in the core region was significantly $(p<0.05)$ higher in the L-PPX group compared to the Sh and Sh-PPX groups. In addition, in the shell region, the $\mathrm{D} 3 \mathrm{R}$ positive cell population was significantly $(p<0.05)$ higher in the L-PPX rats compared to the Sh group.

\section{DISCUSSION}

In this study, we investigated the respective roles of lesion of the VTA and chronic treatment with PPX (32 days) on intermittent daily access to sucrose solution in rats. The main findings are (i)
VTA lesion does not change the amount of sweet solution consumed; (ii) PPX chronic treatment induced in sham and lesioned animals a strong increase of tolerance to sucrose solution, sustained by a strong increase of the amount of sucrose solution drank; (iii) an increase of the dopamine 3 receptor in the shell and the core regions of the nucleus accumbens in animals receiving PPX, with a higher expression in lesioned rats compared to sham; (iv) and finally an increase of anxiety behavior in each group of animals after withdrawal of sucrose access.

When intermittent access to the sweet solution was given to sham animals with saline treatment, we observed an escalation of intake followed by a stable phase. This result is in agreement with previous studies of Avena et al. (2008a,b) showing that animals developed a tolerance for sucrose solution intake when given by intermittent access, followed by a stable phase. Thus, intermittent daily feeding has been described as a model of binge eating in animals since it shares common features with substance dependence such as tolerance and signs of withdrawal (Avena, 2010).

Lesion of the VTA did not change the time-course of sucrose intake. This result is in agreement with studies showing that interference with nucleus accumbens dopamine transmission by depletions or antagonisms has little or no effect on food intake (Salamone and Correa, 2012, 2013) and that 6-OHDA lesion of the VTA has no effect on consumption of sucrose in the $2 \mathrm{~h}$ test period with a two bottle test (Shimura et al., 2002). Indeed, although it is known that modification of dopaminergic innervations from midbrain VTA to forebrain structures, including the nucleus accumbens in the ventral striatum, decreases effortrelated behavior for food, the acquisition of visual-to-sucrose Pavlovian associative learning and development of instrumental goal-directed behavior (Parkinson et al., 2002; Nicola et al., 2005), such manipulation of dopamine leaves the motivation to approach and consume food intact (Salamone and Correa, 2009). Such preservation of motivation could account for the continued sucrose intake escalation observed in lesioned rats. In the context of the intermittent daily feeding experiment, animals did not need to make an association between cues (odor or taste) and sucrose delivery, so they did not need dopamine to trigger associative processes such as "incentive salience attribution" (Berridge and Robinson, 1998). Accordingly, in intermittent daily feeding, it can be assumed that dopamine from the VTA may act only on the initial phase of approach and motivation toward the sucrose solution, while continued escalation could imply other neurotransmitters such as opioids or acetylcholine (Avena et al., 2008a,b) and the action of other brain regions such as the amygdala or prefrontal cortex (Diaz et al., 1995; Hitchcott et al., 1997). An alternative hypothesis is that moderate lesion of VTA, as performed in the present study, leaves compensatory mechanisms intact enough to elicit motivation toward the sucrose solution.

The present data are the first to have been obtained from investigation into the effect of chronic treatment with PPX on day-to-day sucrose intake in animals with lesion of dopaminergic brain system. In both lesioned and sham rats, chronic PPX induced a strong increase of the amount of sucrose solution drank and its ongoing escalation. This result showed that PPX elicited a marked shift in animal tolerance for sucrose. The present results were in agreement with the ones from the study 

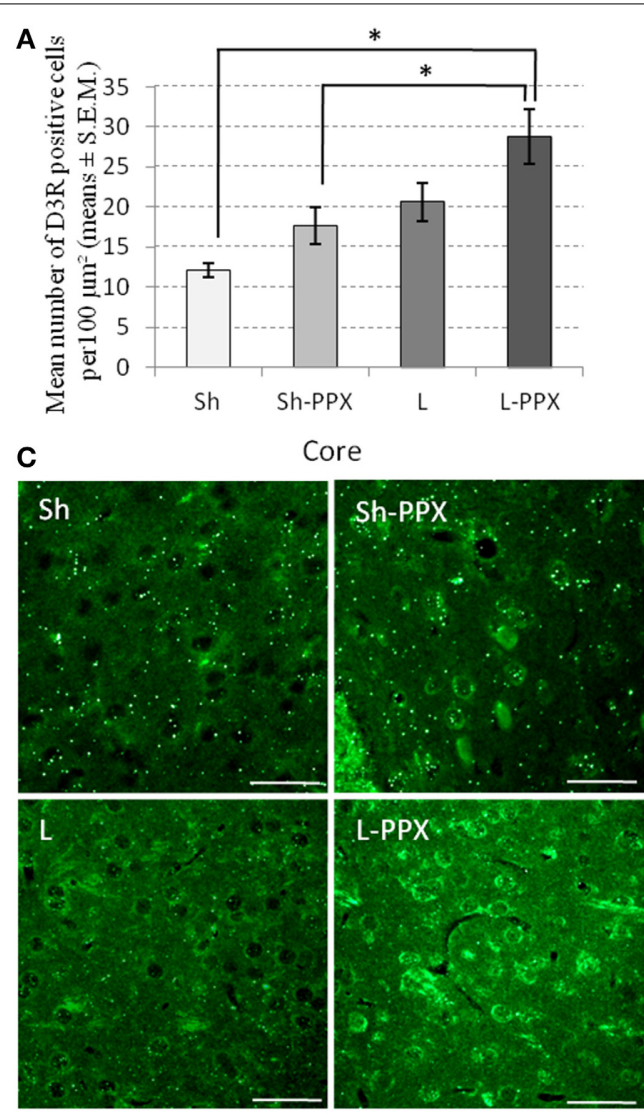

FIGURE 6 | Changes in Dopamine D3 receptor expression following PPX treatment and intermittent access to sucrose solution. In the core region of the accumbens nucleus $(\mathbf{A}, \mathbf{C})$, the number of D3 positive cells was significantly $(* p<0.05)$ higher in L-PPX rats compared to Sh and Sh-PPX ones. Number of D3 positive cells was also significantly $\left({ }^{*} p<0.05\right)$ higher in the shell region of the accumbens nucleus $(\mathbf{B}, \mathbf{D})$ of
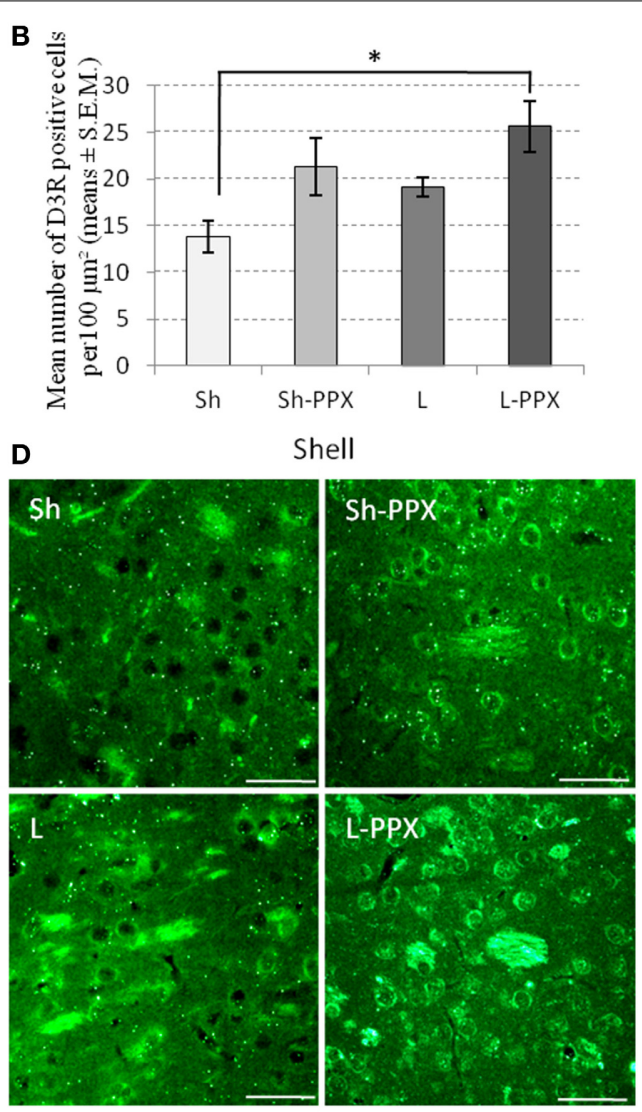

the L-PPX rats compared to Sh ones. Microphotographes are high magnification $(\times 40)$ of the core (C) or the shell (D) regions taken at around $2 \mathrm{~mm}$ from Bregma. White scales bars represent $200 \mu \mathrm{m}$. Sh, Sham animals; Sh-PPX, sham animals with daily pramipexole treatment $(0.1 \mathrm{mg} / \mathrm{kg} / \mathrm{day})$; L, VTA lesioned rats; L-PPX, VTA lesioned rats with daily pramipexole treatment $(0.1 \mathrm{mg} / \mathrm{kg} /$ day $)$. of Willner et al. (1994). They showed that chronic PPX treatment restored $1 \%$ sucrose solution intake in mild stressed rats as well as non-stressed rats. In the case of stressed animals, chronic PPX reversed the stress induced anhedonia, but also induced increase of sucrose intake in normal rats. The role of dopamine in food eating behavior remains controversial in humans and animals, there is strong evidence to suggest that dopamine may participate in food seeking. Indeed, the Dopamine D3 receptor agonist participates in reinstating food seeking at larger doses and potentiates food primed response at low doses (Duarte et al., 2003). In humans, PPX induces weight gain and also restores hunger in PD patients (Kumuru et al., 2006). Furthermore, PD patients under PPX can develop compulsive food eating traits as well as gain weight. Interestingly, when PPX is lowered or discontinued, these symptoms disappear or are reduced (Nirenberg and Waters, 2006). Finally, it has been shown that in overweight and obese subjects, Dopamine D3 receptor antagonists decrease attentional bias toward food cues in low-restrained eaters (Nathan et al., 2012). Thus, the main result of the present work indicate that PPX acts on both lesioned and sham animals by inducing higher tolerance for sweet solutions, pointing to a potential role of D3 receptor stimulation in certain aspects of food-related addiction and compulsion. Such a shift in sucrose intake also seemed to be sustained by molecular modifications in the Acb in the present study.

We showed that D3 receptor expression was higher in animals under chronic treatment compared to those under saline treatment. In addition, we also noted that the number of cells expressing the D3 receptor was significantly higher in the core of the Acb of L-PPX rats compared to Sh-PPX ones. The D3 receptor subtype is mainly expressed in the mesocorticolimbic dopamine pathway (Sokoloff et al., 2001). Recent studies have pointed out that the D3 receptor, which is mostly expressed in the Acb, could play an important role in reinforcement and reward (Heidbreder et al., 2005; Chen et al., 2006). Due to its particular pattern of expression, the D3 receptor has drawn attention due to its potential role in the dysregulation of motivated behaviors in humans (Gyertyán and Gál, 2003; Everitt et al., 2008; Besson et al., 2010) and animals (Engelm et al., 2012). It has been proposed that food anticipation, consumption, and unconditioned consummatory behavior (i.e., eating) activate DA release in their terminal fields, especially in the Acb and prefrontal cortex (Phillips et al., 
1991; Bassareo and Di Chiara, 1997). Such release also seems to be dependent on the reinforcing attraction of available food and on the motivational state of the animal (Wilson et al., 1995; Martel and Fantino, 1996). One possible explanation for our results could be that chronic PPX administration might have amplified the signal associated with the primary reinforcing effect of sucrose presentation in the Acb which contains the D3 receptor at high density, facilitating food-seeking responses. Indeed, chronic PPX administration is known to increase Dopamine D2 and D3 receptor mRNA, D3 dopamine receptor protein and D3 dopamine receptor binding in the striatum and Acb of animals (Maj et al., 2000; Tokunaga et al., 2012).

In this context, the administration of the D3 preferring agonist before the presentation of the sucrose solution, in addition to the dopamine release elicited by the consumption of palatable food (Bassareo and Di Chiara, 1999), could have interfered with the consummatory aspect of food presentation. Such hyperactivation of the dopaminergic system could have positively modulated approach behavior (such as "searching," "wanting") toward the sucrose solution in rats under chronic PPX treatment. However, besides the difference in the pattern of Dopamine D3 receptor positive cells between Sh-PPX and L-PPX, no difference in sucrose tolerance was observed. One possible explanation could be that increased Dopamine D3 receptor expression is only a part of a larger change in brain neurochemistry sustaining tolerance for sucrose. It is known that the lesion of the dopaminergic brain region leads to a profound change in brain neurochemistry and particularly in GABA and glutamate release (Kickler et al., 2009; Chassain et al., 2010, 2013). Such modifications were not explored in the present work and could be addressed in further experiments.

We also noted changes in the expression of anxiety when access to a sweet solution is removed, whatever the experimental groups considered. Such an increase of anxiety in animals after removing access to sucrose has been observed by Hoebel et al. (2007) and Avena et al. (2008b), pointing to opiate-like withdrawal behaviors when sucrose is no longer presented. The change in anxiety may reflect the effect of withdrawal from preferred fluids or drugs that induce negative or aversive states that potentially lead to a relapse of drug consumption (Erb, 2010; Pickens et al., 2011). However, we observed specific changes in anxiety in animals under chronic PPX treatment. L-PPX and, to a lesser extent, Sh-PPX showed increased anxiety compared to their respective controls ( $\mathrm{L}$ and $\mathrm{Sh}$ groups). Such a change in anxiety seems to be linked to dopamine agonist treatment, since the lesion of dopamine system did not modify anxiety in the animal model on its own (Carvalho et al., 2013). The present increase of anxiety in the rats treated could accounts for the higher tolerance for sucrose. Indeed, although the animals were more anxious, they could have been more sensitive to the withdrawal effect observed in the $12 \mathrm{~h}$ period when sucrose was not accessible. Anxiety is one of the factors that contributed to addiction or relapse in humans and rats (Erb, 2010).

Our behavioral results also revealed that neither 1 week of daily treatment by PPX, nor the VTA lesion had an impact on spontaneous locomotor activity and spontaneous alternation in the $\mathrm{Y}$ maze. This result is in agreement with studies showing that lesion of the VTA has no impact on locomotor activity (Pioli et al., 2008; Drui et al., 2013; Ouachikh et al., 2013). The absence of modification of locomotor activity could be also due to the PPX dose chosen in the present experiment. We used a dose of $0.1 \mathrm{mg} / \mathrm{kg}$ based on the study of Collins et al. (2005), showing that such a low dose induced preferential activation of the D3 receptor. In addition, this low dose induced brief hypolocomotion after injection (30 min periods) without inducing hyperlocomotion (Chang et al., 2011). In the present work, in order to avoid a confounding effect of PPX on locomotion, we subjected animals to behavioral tasks at least $2 \mathrm{~h}$ after PPX injection. The fact that the animals did not display disturbed locomotion with lesions or with PPX treatment allowed us to validate our results for the other behavioral tasks.

To conclude, the present results showed that long-term stimulation of the D3 receptor using PPX induced an increase in sucrose solution intake as well as in the rate of escalation in sham animals and lesioned rats, along with an increase of the expression of the D3 receptor in the ACb. Despite an increase of D3 receptor expression in Acb compared to sham PPX, no change of sucrose solution intake was observed in lesioned animals receiving PPX, thus showing the main role played by PPX in this behavior compared to the VTA lesion itself.

\section{ACKNOWLEDGMENTS}

Dr. David Dardou planned, performed, and analyzed all the experiments and wrote the manuscript. Dr. Carine Chassain participated in the immunohistochemical experiments and analysis and revised the manuscript. Dr. Franck Durif participated in writing and revising the manuscript. The authors thank G. Keleva for her helpful assistance in the immunohistochemistry experiments.

\section{REFERENCES}

Avena, N. M. (2010). The study of food addiction using animal models of binge eating. Appetite 55, 734-737. doi: 10.1016/j.appet.2010.09.010

Avena, N. M., Bocarsly, M. E., Rada, P., Kim, A., and Hoebel, B. G. (2008b) After daily bingeing on a sucrose solution, food deprivation induces anxiety and accumbens dopamine/acetylcholine imbalance. Physiol. Behav. 94, 309-315. doi: 10.1016/j.physbeh.2008.01.008

Avena, N. M., Rada, P., and Hoebel, B. G. (2008a). Underweight rats have enhanced dopamine release and blunted acetylcholine response in the nucleus accumbens while bingeing on sucrose. Neuroscience 156, 865-871. doi: 10.1016/j.neuroscience.2008.08.017

Bassareo, V., and Di Chiara, G. (1997). Differential influence of associative and nonassociative learning mechanisms on the responsiveness of prefrontal and accumbal dopamine transmission to food stimuli in rats fed ad libitum. J. Neurosci. 17, 851-861.

Bassareo, V., and Di Chiara, G. (1999). Modulation of feeding-induced activation of mesolimbic dopamine transmission by appetitive stimuli and its relation to motivational state. Eur. J. Neurosci. 11, 4389-4397. doi: 10.1046/j.14609568.1999.00843.x

Berridge, K. C., and Robinson, T. E. (1998). What is the role of dopamine in reward: hedonic impact, reward learning, or incentive salience? Brain Res. Rev. 28, 309-369. doi: 10.1016/S0165-0173(98)00019-8

Besson, M., Belin, D., McNamara, R., Theobald, D. E., Castel, A., Beckett, V. L., et al. (2010). Dissociable control of impulsivity in rats by dopamine $\mathrm{d} 2 / 3$ receptors in the core and shell subregions of the nucleus accumbens. Neuropsychopharmacology 35, 560-569. doi: 10.1038/npp.2009.162

Bordet, R., Ridray, S., Schwartz, J. C., and Sokoloff, P. (2000). Involvement of the direct striatonigral pathway in levodopa-induced sensitization in 6-hydroxydopamine-lesioned rats. Eur. J. Neurosci. 12, 2117-2123. doi: 10.1046/j.1460-9568.2000.00089.x 
Carvalho, M. M., Campos, F. L., Coimbra, B., Pêgo, J. M., Rodrigues, C., Lima, R., et al. (2013). Behavioral characterization of the 6-hydroxidopamine model of Parkinson's disease and pharmacological rescuing of non-motor deficits. Mol. Neurodegener. 8, 14-25. doi: 10.1186/1750-1326-8-14

Chang, W. L., Breier, M. R., Yang, A., and Swerdlow, N. R. (2011). Disparate effects of pramipexole on locomotor activity and sensorimotor gating in Sprague-Dawley rats. Pharmacol. Biochem. Behav. 99, 634-638. doi: 10.1016/j.pbb.2011.06.002

Chassain, C., Bielicki, G., Carcenac, C., Ronsin, A. C., Renou, J. P., Savasta, M., et al. (2013). Does MPTP intoxication in mice induce metabolite changes in the nucleus accumbens? A ${ }^{1} \mathrm{H}$ nuclear MRS study. NMR Biomed. 26, 336-347. doi: $10.1002 / \mathrm{nbm} .2853$

Chassain, C., Bielicki, G., Keller, C., Renou, J. P., and Durif, F. (2010). Metabolic changes detected in vivo by $1 \mathrm{H}$ MRS in the MPTP-intoxicated mouse. NMR Biomed. 23, 547-553. doi: 10.1002/nbm.1504

Chen, G., Kittler, J. T., Moss, S. J., and Yan, Z. (2006). Dopamine D3 receptors regulate GABA receptor function through a phospho-dependent endocytosis mechanism in nucleus accumbens. J. Neurosci. 26, 2513-2521. doi: 10.1523/JNEUROSCI.4712-05.2006

Collins, G. T., Witkin, J. M., Newman, A. H., Svensson, K. A., Grundt, P., Cao, J., et al. (2005). Dopamine agonist-induced yawning in rats: a dopamine D3 receptor-mediated behavior. J. Pharmacol. Exp. Ther. 314, 310-319. doi: 10.1124/jpet.105.085472

Courty, E., Durif, F., Zenut, M., Courty, P., and Lavarenne, J. (1997). Psychiatric and sexual disorders induced by apomorphine in Parkinson's disease. Clin. Neuropharmacol. 20, 140-147. doi: 10.1097/00002826-19970400000005

Dagher, A., and Robbins, T. W. (2009). Personality, addiction, dopamine: insights from Parkinson's disease. Neuron 61, 502-510. doi: 10.1016/j.neuron.2009.01.031

Diaz, J., Lévesque, D., Lammers, C. H., Griffon, N., Martres, M. P., Schwartz, J. C., et al. (1995). Phenotypical characterization of neurons expressing the dopamine D3 receptor in the rat brain. Neuroscience 65, 731-745. doi: 10.1016/03064522(94)00527-C

Drui, G., Carnicella, S., Carcenac, C., Favier, M., Bertrand, A., Boulet, S., et al. (2013). Loss of dopaminergic nigrostriatal neurons accounts for the motivational and affective deficits in Parkinson's disease. Mol. Psychiatry 19, 358-367. doi: $10.1038 / \mathrm{mp} .2013 .3$

Duarte, C., Lefebvre, C., Chaperon, F., Hamon, M., and Thiébot, M. H. (2003). Effects of a dopamine D3 receptor ligand, BP 897, on acquisition and expression of food-, morphine-, and cocaine-induced conditioned place preference, and food-seeking behavior in rats. Neuropsychopharmacology 28, 1903-1915. doi: 10.1038/sj.npp.1300276

Engelm, M., Ahmed, S. H., Vouillac, C., Tison, F., Bezard, E., and Fernagut, P. O. (2012). Reinforcing properties of Pramipexole in normal and parkinsonian rats. Neurobiol. Dis. 49C, 79-86. doi: 10.1016/j.nbd.2012.08.005

Erb, S. (2010). Evaluation of the relationship between anxiety during withdrawal and stress-induced reinstatement of cocaine seeking. Prog. Neuropsychopharmacol. Biol. Psychiatry 34, 798-807. doi: 10.1016/j.pnpbp.2009.11.025

Everitt, B. J., Belin, D., Economidou, D., Pelloux, Y., Dalley, J. W., and Robbins, T. W. (2008). Neural mechanisms underlying the vulnerability to develop compulsive drug-seeking habits and addiction. Philos. Trans. R. Soc. Lond. B Biol. Sci. 363, 3125-3135. doi: 10.1098/rstb.2008.0089

Gallagher, G. A., O’Sullivan, S. S., Evans, A. H., Lees, A. J., and Schrag, A. (2007). Pathological gambling in Parkinson's disease: risk factors and differences from dopamine dysregulation. An analysis of published case series. Mov. Disord. 22, 1757-1763. doi: 10.1002/mds.21611

German, D. C., Manaye, K., Smith, W. K., Woodward, D. J., and Saper, C. B. (1989). Midbrain dopaminergic cell loss in Parkinson's disease: computer visualization. Ann. Neurol. 26, 507-514. doi: 10.1002/ana.410260403

Gyertyán, I., and Gál, K. (2003). Dopamine D3 receptor ligands show place conditioning effect but do not influence cocaine-induced place preference. Neuroreport 14, 93-98. doi: 10.1097/00001756-20030120000018

Heidbreder, C. A., Gardner, E. L., Xi, Z. X., Thanos, P. K., Mugnaini, M., Hagan, J. J., et al. (2005). The role of central dopamine D3 receptors in drug addiction: a review of pharmacological evidence. Brain Res. Rev. 49, 77-105. doi: 10.1016/j.brainresrev.2004.12.033
Hitchcott, P. K., Bonardi, C. M., and Phillips, G. D. (1997). Enhanced stimulusreward learning by intra-amygdala administration of a D3 dopamine receptor agonist. Psychopharmacology (Berl.) 133, 240-248. doi: 10.1007/s002130 050397

Hoebel, B. G., Avena, N. M., and Rada, P. (2007). Accumbens dopamineacetylcholine balance in approach and avoidance. Curr. Opin. Pharmacol. 7, 617-627. doi: 10.1016/j.coph.2007.10.014

Kickler, N., Lacombe, E., Chassain, C., Durif, F., Krainik, A., Farion, R., et al. (2009). Assessment of metabolic changes in the striatum of a rat model of parkinsonism: an in vivo (1)H MRS study. NMR Biomed. 22, 207-212. doi: 10.1002/nbm.1305

Kumuru, H., Santamaria, J., Valldeoriola, F., Marti, M. J., and Tolosa, E. (2006). Increase in body weight after pramipexole treatment in Parkinson's disease. Mov. Disord. 21, 1972-1974. doi: 10.1002/mds.21086

Le Foll, B., Goldberg, S. R., and Sokoloff, P. (2005). The dopamine D3 receptor and drug dependence: effects on reward or beyond? Neuropharmacology 49, 525-541. doi: 10.1016/j.neuropharm.2005.04.022

Maj, J., Rogói, Z., Margas, W., Kata, M., and Dziedzicka-Wasylewska, M. (2000). The effect of repeated treatment with pramipexole on the central dopamine D3 system. J. Neural Transm. 107, 1369-1379. doi: 10.1007/s007020070001

Martel, P., and Fantino, M. (1996). Mesolimbic dopaminergic system activity as a function of food reward: a microdialysis study. Pharmacol. Biochem. Behav. 53, 221-226. doi: 10.1016/0091-3057(95)00187-5

Merims, D., and Giladi, N. (2008). Dopamine dysregulation syndrome, addiction and behavioral changes in Parkinson's disease. Parkinsonism Relat. Disord. 14, 273-280. doi: 10.1016/j.parkreldis.2007.09.007

Miwa, H., and Kondo, T. (2008). Alteration of eating behaviors in patients with Parkinson's disease: possibly overlooked? Neurocase 14, 480-484. doi: 10.1080/13554790802495324

Mugani, M., Iavarone, L., Cavallini, P., Griffante, C., Oliosi, B., Savoia, C., et al. (2013). Occupancy of brain dopamine D3 receptor and drug craving: a translational approach. Neuropsychopharmacology 38, 301-312. doi: 10.1038/npp.2012.171

Nathan, P. J., O’Neill, B. V., Mogg, K., Bradley, B. P., Beaver, J., Bani, M. et al. (2012). The effects of the dopamine D3 receptor antagonist GSK598809 on attentional bias to palatable food cues in overweight and obese subjects. Int. J. Neuropsychopharmacol. 15, 149-161. doi: 10.1017/S1461145711 001052

Nicola, S. M., Taha, S. A., Kim, S. W., and Fields, H. L. (2005). Nucleus accumbens dopamine release is necessary and sufficient to promote the behavioral response to reward-predictive cues. Neuroscience 135,1025-1033. doi: 10.1016/j.neuroscience.2005.06.088

Nirenberg, M. J., and Waters, C. (2006). Compulsive eating and weight gain related to dopamine agonist use. Mov. Disord. 21, 524-529. doi: 10.1002/mds. 20757

Ouachikh, O., Dieb, W., Durif, F., and Hafidi, A. (2013). Differential behavioral reinforcement effects of dopamine receptor agonists in the rat with bilateral lesion of the posterior ventral tegmental area. Behav. Brain Res. 252, 24-31. doi: 10.1016/j.bbr.2013.05.042

Ouachikh, O., Dieb, W., Durif, F., and Hafidi, A. (2014). Anterior ventral tegmental area dopaminergic neurons are not involved in the motivational effects of bromocriptine, pramipexole and cocaine in drug-free rats. Behav. Brain Res. 262, 1-7. doi: 10.1016/j.bbr.2013.12.021

Paillé, V., Henry, V., Lescaudron, L., Brachet, P., and Damier, P. (2007). Rat model of Parkinson's disease with bilateral motor abnormalities, reversible with levodopa, and dyskinesias. Mov. Disord. 22, 533-539. doi: 10.1002/mds.21308

Papp, M. (1988). Different effects of short- and long-term treatment with imipramine on the apomorphine- and food-induced place preference conditioning in rats. Pharmacol. Biochem. Behav. 30, 889-893. doi: 10.1016/00913057(88)90115-3

Parkinson, J. A., Dalley, J. W., Cardinal, R. N., Bamford, A., Fehnert, B., Lachenal, G., et al. (2002). Nucleus accumbens dopamine depletion impairs both acquisition and performance of appetitive Pavlovian approach behavior: implications for mesoaccumbens dopamine function. Behav. Brain Res. 137, 149-163. doi: 10.1016/S0166-4328(02)00291-7

Phillips, G., Willner, P., and Muscat, R. (1991). Reward-dependent suppression or facilitation of consummatory behaviour by raclopride. Psychopharmacology (Berl.) 105, 355-360. doi: 10.1007/BF02244430

Pickens, C. L., Airavaara, M., Theberge, F., Fanous, S., Hope, B. T., and Shaham, Y. (2011). Neurobiology of the incubation of drug craving. Trends Neurosci. 34, 411-420. doi: 10.1016/j.tins.2011.06.001 
Pioli, E. Y., Meissner, W., Sohr, R., Gross, C. E., Bezard, E., and Bioulac, B. H. (2008). Differential behavioral effects of partial bilateral lesions of ventral tegmental area or substantia nigra pars compacta in rats. Neuroscience 153, 1213-1224. doi: 10.1016/j.neuroscience.2008.01.084

Rice, O. V., Heidbreder, C. A., Gardner, E. L., Schonbar, C. D., and Ashby Fr, C. R. (2013). The selective D3 receptor antagonist SB-277011A attenuates morphine triggered reactivation of expression of cocaine-induced conditioned place preference. Synapse 67, 469-475. doi: 10.1002/syn. 21653

Riddle, J. L., Rokosik, S. L., and Napier, T. C. (2012). Pramipexole- and methamphetamine-induced reward-mediated behavior in a rodent model of Parkinson's disease and controls. Behav. Brain Res. 233, 15-23. doi: 10.1016/j.bbr.2012.04.027

Rieu, I., Derost, P., Ulla, M., Marques, A., Debilly, B., De Charenzon, I., et al. (2011). Body weight gain and deep brain stimulation. J. Neurol. Sci. 310, 267-270. doi: 10.1016/j.jns.2011.06.055

Robinson, T. E., and Berridge, K. C. (2001). Incentive-sensitization and addiction. Addiction 96, 103-114. doi: 10.1046/j.1360-0443.2001. 9611038.x

Salamone, J. D., and Correa, M. (2009). Dopamine/adenosine interactions involved in effort-related aspects of food motivation. Appetite 53, 422-425. doi: 10.1016/j.appet.2009.07.018

Salamone, J. D., and Correa, M. (2012). The mysterious motivational functions of mesolimbic dopamine. Neuron 76, 470-485. doi: 10.1016/j.neuron.2012. 10.021

Salamone, J. D., and Correa, M. (2013). Dopamine and food addiction: lexicon badly needed. Biol. Psychiatry 73, e15-e24. doi: 10.1016/j.biopsych.2012. 09.027

Shimura, T., Kamada, Y., and Yamamoto, T. (2002). Ventral tegmental lesions reduce overconsumption of normally preferred taste fluid in rats. Behav. Brain Res. 134, 123-130. doi: 10.1016/S0166-4328(01)00461-2

Sokoloff, P., Le Foll, B., Perachon, S., Bordet, R., Ridray, S., and Schwartz, J. C. (2001). The dopamine D3 receptor and drug addiction. Neurotox. Res. 3, 433-441. doi: 10.1007/BF03033202

Taghzouti, K., Simon, H., and Le Moal, M. (1986). Disturbances in exploratory behavior and functional recovery in the $\mathrm{Y}$ and radial mazes following dopamine depletion of the lateral septum. Behav. Neural Biol. 45, 48-56. doi: 10.1016/S0163-1047(86)80005-X

Thobois, S., Ardouin, S., Lhommée, E., Klinger, H., Lagrange, C., Xie, J., et al. (2010). Non-motor dopamine withdrawal syndrome after surgery for Parkinson's disease: predictors and underlying mesolimbic denervation. Brain 133, 1111-1127. doi: 10.1093/brain/awq032
Thobois, S., Lhommée, E., Klinger, H., Ardouin, C., Schmitt, E., Bichon, A., et al. (2013). Parkinsonian apathy responds to dopaminergic stimulation of D2/D3 receptors with piribedil. Brain 136, 1568-1577. doi: 10.1093/brain/awt067

Tokunaga, N., Choudhury, M. E., Nishikawa, N., Nagai, M., Tujii, T., Iwaki, H., et al. (2012). Pramipexole upregulates dopamine receptor D2 and D3 expression in rat striatum. J. Phamacol. Sci. 120, 133-137. doi: 10.1254/jphs.12096SC

Voon, V., and Fox, S. H. (2007). Medication-related impulse control and repetitive behaviors in Parkinson disease. Arch. Neurol. 64, 1089-1096. doi: 10.1001/archneur.64.8.1089

Weintraub, D., Koester, J., Potenza, M. N., Siderowf, A. D., Stacy, M., Voon, V., et al. (2010). Impulse control disorders in Parkinson disease: a cross-sectional study of 3090 patients. Arch. Neurol. 67, 589-595. doi: 10.1001/archneurol.2010.65

Willner, P., Lappas, S., Cheeta, S., and Muscat, R. (1994). Reversal of stress-induced anhedonia by the dopamine receptor agonist, pramipexole. Psychopharmacology (Berl.) 115, 454-462. doi: 10.1007/BF02245568

Wilson, C., Nomikos, G. G., Collu, M., and Fibiger, H. C. (1995). Dopaminergic correlates of motivated behavior: importance of drive. J. Neurosci. 15, 5169-5178.

Zengin-Toktas, Y., Authier, N., Denizot, H., Chassain, C., Hafidi, A., Llorca, P. M., et al. (2013). Motivational properties of D2 and D3 dopamine receptors agonists and cocaine, but not with D1 dopamine receptors agonist and Ldopa, in bilateral 6-OHDA lesioned rat. Neuropharmacology 70, 74-82. doi: 10.1016/j.neuropharm.2012.12.011

Conflict of Interest Statement: : Dr. Franck Durif receives remuneration as a consultant for Novartis, Teva, Lundback, UCB, Aguettant, and Allergan. The authors declare that the research was conducted in the absence of any commercial or financial relationships that could be construed as a potential conflict of interest.

Received: 02 September 2014; accepted: 11 December 2014; published online: 06 January 2015.

Citation: Dardou D, Chassain C and Durif F (2015) Chronic pramipexole treatment increases tolerance for sucrose in normal and ventral tegmental lesioned rats. Front. Neurosci. 8:437. doi: 10.3389/fnins.2014.00437

This article was submitted to Neuropharmacology, a section of the journal Frontiers in Neuroscience.

Copyright (C) 2015 Dardou, Chassain and Durif. This is an open-access article distributed under the terms of the Creative Commons Attribution License (CC BY). The use, distribution or reproduction in other forums is permitted, provided the original author(s) or licensor are credited and that the original publication in this journal is cited, in accordance with accepted academic practice. No use, distribution or reproduction is permitted which does not comply with these terms. 